\title{
WHAT ARE THE GAP BETWEEN GERIATRICIANS AND ONCOLOGIST? A SURVEY OF DOCTOR AWARENESS OF MEDICAL CARE AMONG ELDERLY PATIENTS WITH CANCER IN SOUTHWEST CHINA
}

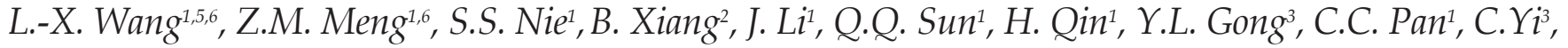 \\ Y.X. Yang ${ }^{5}$ S. Wang
}

\begin{abstract}
Objectives: Old patients with cancer are a great challenge to physicians. This study investigated the similarities and differences in the respective opinions of geriatricians and oncologists with regard to clinical practice among elderly patients with cancer in south west China. Design, Setting, Participants and Measurements: Doctors from five local public hospitals of Sichuan were surveyed with a questionnaire via face-to-face interview. The ratio of geriatricians to oncologists' was1:1. The questionnaire addressed respondents' demographic characteristics and was composed of 11 multiple-choice questions and a clinical scenario. Results: The respondents included 64 oncologists and geriatricians, respectively. More than forty percent participants believed that current treatments were under-treatment. "Opinion of family members» was the top one reason for under-treatment in both groups, more oncologist choice «comorbidity» as cause of insufficient treatment than geriatricians ( $\mathrm{p}=0.001)$. Age $(57.03 \%)$ was the fifth effect factor impacting on treatment decision except for physical function (81.25\%), comorbidity (71.09\%) and cancer itself. Physical function, comorbidity and age between 65-84, geriatrician and oncologist did have different attitude. Conclusions: The attitude to physical function and comorbidity were the main difference between geriatrician and oncologist for their clinical decisions. A workshop should be set up to optimize the treatment proceedings.
\end{abstract}

Key words: Physicians' awareness, medical care of old cancer patient, geriatrician, oncologist, questionnaire survey.

\section{Introduction}

The incidence of cancer increases with age. Because of comorbidity and the declining reserves of physical function, doctors should consider additional factors when developing anti-tumor treatment regimens for elderly cancer patients (1). Although some countries have established a multidisciplinary working group according to suggestions of the International Society of Geriatric Oncology (SIOG) (2), in China, the cooperation between geriatricians and oncologists is insufficient. Using a questionnaire, the current study investigated the treatment-related factors and decision-making attitudes

1. The Center of Gerontology and Geriatrics, West China Hospital, Sichuan University, Sichuan, China; Current address: The Fifth People's Hospital of Chengdu, Sichuan, China; 2. The Department of Hematology, West China Hospital, Sichuan University, Sichuan, China; 3. The Center of Cancer, West China Hospital, Sichuan University, Sichuan, China; 4. The Sichuan Cancer Hospital, Sichuan, China; 5. The Fifth People's Hospital of Chengdu, Sichuan, China; 6. Those two authors contribute equal to this work.

Corresponding Author: Shuang Wang, MD, the Center of Gerontology and Geriatrics, West China Hospital, Sichuan University. 37 Guoxuexiang, Chengdu, Sichuan providence, PR. China, 610041; Tel: 86-28-85423239; Fax: 86-28-85422321; E-mail: wangs0211@hotmail.com among oncologists and geriatricians. The results of this study are expected to outline the current knowledge gaps between the two specialties.

\section{Participants and Methods}

\section{Participants}

Licensed physicians employed by the Geriatrics Center, Cancer Center and Department of Hematology at West China Hospital, Sichuan University; the Department of Geriatrics at the Sichuan Cancer Hospital and the Fifth People's Hospital of Chengdu were involved. The questionnaire was administered in December 2013.

\section{Methods}

A face-to-face interview method was adopted for the survey. The questionnaire (see Appendix) was designed based on a review of the extant literature (1). Patients with one or more of the following were recognized as 
Table 1

General respondent information

\begin{tabular}{|c|c|c|c|c|}
\hline & Total $(n=128)$ & Geriatrician $(n=64)$ & Oncologist $(n=64)$ & P-value \\
\hline Gender (female) & $90(70.31 \%)$ & $46(71.87 \%)$ & $44(68.75 \%)$ & .699 \\
\hline Proportion of elderly cancer patients & & & & .079 \\
\hline$<10 \%$ & $22(17.19 \%)$ & $5(7.81 \%)$ & $10(15.63 \%)$ & -- \\
\hline $10 \%-30 \%$ & $30(23.44 \%)$ & $12(18.75 \%)$ & $16(25 \%)$ & \\
\hline $50 \%-70 \%$ & $45(35.16 \%)$ & $27(42.19 \%)$ & $18(28.13 \%)$ & \\
\hline$>70 \%$ & $5(3.9 \%)$ & $14(21.88 \%)$ & 0 & \\
\hline Previous cooperation with a geriatrician & -- & -- & $21(32.81 \%)$ & -- \\
\hline Previous use of any kind of geriatric assessment tool & 25 & 20 & 5 & \\
\hline In most cases & -- & -- & $11(52.38 \%)$ & \\
\hline Always & -- & -- & $5(23.81 \%)$ & \\
\hline
\end{tabular}

${ }^{*} \mathrm{n}=21$; the number of oncologists who had cooperated with a geriatrician

having geriatric syndrome: more than one dependence of actively of daily living (ADL, including dressing, eating, ambulating, toileting and hygiene) and/or instrument of ADL (IADL, including shopping, housework, accounting, food preparation and transportation); Comorbidity; mental/cognitive impairment; mood disorder; visual/ hearing impairments; and malnutrition. Comorbidity means a state with the simultaneous appearance of two or more psychiatric or physical illnesses.

The content of the questionnaire included patient demographic information, 11 multiple-choice questions, and a clinical scenario. The 11 multiple-choice questions cover the following: 1) understanding and applying the knowledge of geriatric syndrome in clinical practice; 2 ) the effect of other factors (such as caregiver availability or family economy and environment) on treatment decisions for elderly cancer patients; and 3) the doctors' acceptance of the multidisciplinary management of elderly cancer patients. A simple clinical scenario was designed to test the extent to which chronologic age per se effected the treatment decision.

The pre-survey portion of the questionnaire was conducted at the Geriatrics Center and the Cancer Center of West China Hospital, Sichuan University. Four oncologists and four geriatricians with different years of work experience (0-5 years, 5-10 years, 10-20 years, or more than 20 years working at a subspecialty clinic after obtaining a Bachelor of Medicine Degree) were selected from the above departments to complete the survey. The questionnaire was modified based on the results of the pre-survey. All investigators received unified training for the administration of the questionnaire, without knowledge of its design or purpose.
The formal survey was first conducted among the geriatricians at the Geriatrics Center of West China Hospital, Sichuan University and the elderly ward of the Fifth People's Hospital in Chengdu. Using a 1:1 matching ratio, oncologists with the corresponding amount of work experience (0-5 years, 5-10 years, 10-20 years, or more than 20 years working at a subspecialty clinic after obtaining a Bachelor's Degree) were selected from the Cancer Center and the Department of Hematology at West China Hospital, Sichuan University and the Department of Oncology at the Cancer Hospital of Sichuan Province to complete the survey via a face-toface interview.

The investigators answered all questions raised by the respondents during the questionnaire administration on site. Questionnaires with incomplete answers were considered invalid.

\section{Statistical analyses}

Descriptive statistics included the mean, median, and proportion. The difference in the age of the doctors between the two specialties was compared using a t-test. The between-specialty differences were analyzed using the $\chi 2$-test. Differences with P-values $\leq .05$ were considered significant.

\section{Results}

\section{General respondent information}

A total of 148 questionnaires were distributed, and 191

$128(86.5 \%)$ valid questionnaires were collected. The 
Table 2

Current treatment status and the factors influencing treatment decisions

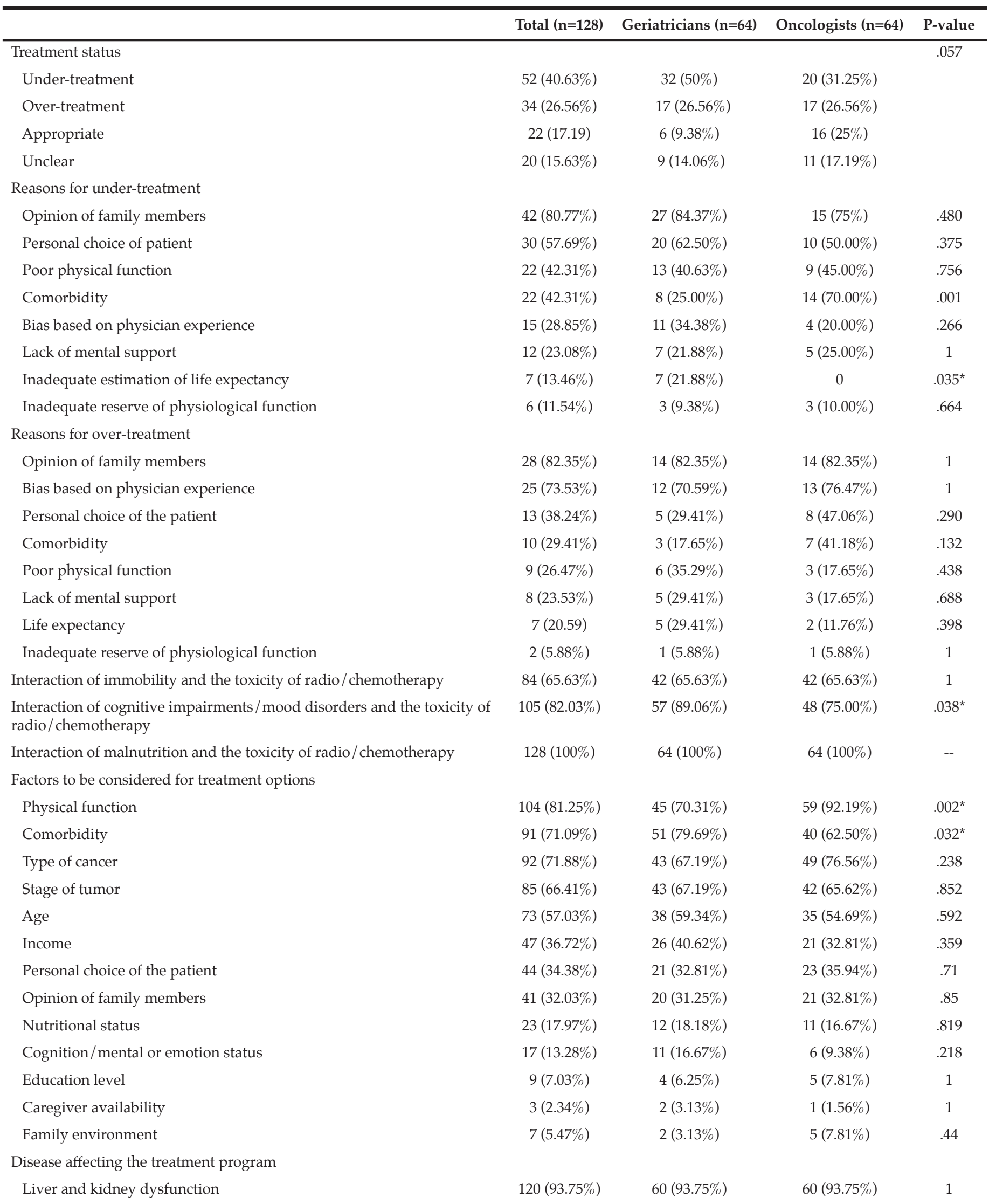




Chronic heart failure
COPD or other chronic respiratory diseases
Coronary heart disease
Digestive system diseases
Transient ischemic attack (TIA) or cerebral infarction
Type 2 diabetes
Dementia
Hypertension
Moderate-to-severe osteoporosis
The greatest difficulty when deciding upon a treatment plan
Lack of expertise in geriatrics
Patients used to trust the oncologist more
Frailty among elderly patients
Multidisciplinary management is feasible
Note:*indicates p<.05; \#ndicates p<.001
respondents' general information and values for the
degree of their mutual cooperation are listed in Table
1. The mean age of the respondents was 36 years $(25-$
53). The age, gender, and proportion of elderly cancer
patients in their practice were all comparable. The mean
proportion of elderly patients treated by oncologists
was $59.38 \%$ In addition, $32.81 \%$ of the oncologists had
worked with a geriatrician before.
and on

Up to $70 \%$ of the geriatricians and $57 \%$ of the oncologists believed that the current treatments for elderly cancer patients were inappropriate (underand over-treatment were considered inappropriate treatments). Relatively, more oncologists $(25 \%)$ than geriatricians $(9.38 \%)$ thought current treatments were appropriate. Among them, 50\% of the geriatricians and $31.25 \%$ of the oncologists believed that current treatments were inadequate. Although $70 \%$ of the oncologists believed that comorbidity was the cause of under-treatment, only $25 \%$ of the geriatricians agreed with this opinion ( $\mathrm{p}=0.001)$. «Opinion of family members» was considered to be the primary reason for both under- $(80.77 \%)$ and over-treatment $(82.35 \%)$ by both specialties. As for reasons for over-treatment, no difference was found between the two groups. Regardless of specialty, all doctors were concerned about the interactions between the toxic side effects of radioand chemotherapy and geriatric symptoms, such as malnutrition $(100 \%)$, poor physical function $(65.63 \%)$, and cognitive impairments/mood disorders (geriatricians vs. oncologists: $89.6 \%$ vs. $75 \%$ ).

Regarding the factors considered in treatment

\begin{tabular}{lccc}
$115(89.84 \%)$ & $58(90.63 \%)$ & $57(89.06 \%)$ & .77 \\
$90(70.31 \%)$ & $36(56.25 \%)$ & $54(84.38 \%)$ & $.000 \#$ \\
$78(60.94 \%)$ & $38(59.38 \%)$ & $40(62.50 \%)$ & .717 \\
$60(46.88 \%)$ & $33(51.56 \%)$ & $27(42.19 \%)$ & .288 \\
$48(37.5 \%)$ & $24(37.50 \%)$ & $24(37.50 \%)$ & 1 \\
$46(35.94 \%)$ & $26(40.63 \%)$ & $20(31.25 \%)$ & .269 \\
$40(31.25 \%)$ & $21(32.81 \%)$ & $19(29.69 \%)$ & .703 \\
$26(20.31 \%)$ & $10(25.635)$ & $16(25.00 \%)$ & .287 \\
$15(11.72 \%)$ & $12(18.75 \%)$ & $3(4.69 \%)$ & $.013^{*}$ \\
$88(68.75 \%)$ & $40(62.50 \%)$ & $48(75.00 \%)$ & .127 \\
$59(46.09 \%)$ & $37(57.81 \%)$ & $22(34.38 \%)$ & $.008^{*}$ \\
$21(16.41 \%)$ & $4(6.06 \%)$ & $17(26.56 \%)$ & $.002^{*}$ \\
$128(100 \%)$ & $64(100 \%)$ & $64(100 \%)$ & -- \\
\hline
\end{tabular}

decisions, physical function $(81.25 \%)$, comorbidity $(71.09 \%)$, cancer type $(71.88 \%)$, tumor stage $(66.41 \%)$, and patient age $(57.03 \%)$ were thought to be the top five considerations. More oncologists (92.19\%) were concerned with physical function than geriatricians $(70.31 \%)$. As for comorbidity, liver/kidney dysfunction $(93.75 \%)$ and chronic heart failure $(89.84 \%)$ were more concerning to the doctors, regardless of specialty. More oncologists paid attention to chronic obstructive pulmonary disease (COPD) than geriatricians $(84.38 \%$ and $56.25 \% ; \mathrm{p}<0.001)$. However, more geriatricians $(57.81 \%)$ believed that "patients tended to trust oncologists more", causing difficulties in managing elderly cancer patients, whereas only $34.38 \%$ of oncologist agreed $(\mathrm{p}=0.008)$.

All respondents $(100 \%)$ agreed that the establishment of a multidisciplinary management model for elderly cancer patients is feasible (Table 2).

\section{Effect of chronologic age on the treatment decision}

Even if the patient was a "healthy" person, $32.81 \%$ of oncologists believed that an adjusted therapy should be given to 65-74 year-old cancer patients $(p=0.003)$, which is higher than the geriatricians $(10.94 \%)$; almost one third of oncologists choose a supportive therapy for 75-84 yearold patients $(p=0.010)$, which is more than the number of geriatricians recommending this approach $(12.5 \%)$. All doctors chose to provide supportive therapy to patients older than 85 years (Table 3 ).

\section{Discussion}

Compared with younger patients, elderly cancer patients are more diverse and complex with regard to their physical, psychological, and socioeconomic conditions. According to our results, many doctors in 
Table 3

A clinical scenario

\begin{tabular}{|c|c|c|c|c|}
\hline Age & & Geriatrician $(n=64)$ & Oncologist $(n=64)$ & P-value \\
\hline \multirow{3}{*}{ 65-74 years } & Standard therapy & $57(89.06 \%)$ & $43(67.19 \%)$ & \\
\hline & Adjusted therapy & $7(10.94 \%)$ & $21(32.81 \%)$ & \\
\hline & Supportive therapy & 0 & 0 & \\
\hline \multirow{2}{*}{ 75-84 years } & Adjusted therapy & $53(82.81 \%)$ & $41(64.06 \%)$ & .016 \\
\hline & Supportive therapy & $8(12.5 \%)$ & $20(31.25 \%)$ & .010 \\
\hline \multirow[t]{2}{*}{$\geq 85$ years } & & & & -- \\
\hline & Standard therapy & 0 & 0 & \\
\hline
\end{tabular}

southwestern China believe that the current treatment of elderly cancer patients in Sichuan is either under- or over-treatment, which is similar to other reports $(3,4)$ in this field. A lack of clinical evidence is partly responsible for inadequate treatment among the elderly because these patients (especially those older than 70 years, even if they are healthy) are often excluded from clinical studies (1). Therefore, managing the care of elderly cancer patients properly is a significant worldwide challenge to both geriatricians and oncologists. Interestingly, our survey found that the "opinion of family members» was the primary cause of inadequate or excessive treatment from the physician's point of view. This finding might be a culture-specific result, because in China, family members have always played important roles. Especially when the elderly are suffering from cancer, the family tends to conceal the truth from the patient.

Our survey also showed that comorbidity, physical function, cancer type, tumor stage, and patient age were the top five factors for both geriatricians and oncologists in southwestern China when they made their clinical decisions. Among them, physical function was the most common factor that was viewed as important by both oncologists and geriatricians. This result revealed that for the majority of physicians evaluating functional status, there is no knowledge gap between oncologists and geriatricians in southwestern China. The significant difference was that geriatricians valued the interaction between cognitive impairment/mood disorders and the toxicity of radiation and chemotherapy more than oncologists, and they were more concerned about the comorbidity of dementia, whereas the oncologists were concerned about COPD. The geriatricians believed that patients trust the oncologist more than the geriatrician, whereas the oncologists did not hold this belief. Our survey also found that most of the participants lacked a systematic assessment tool (such as a geriatric assessment, GA). In some studies, GA-guided treatment plans have been shown to improve overall survival, quality of life, and physical function and decrease the risk of hospitalization in the geriatric population. However, data on the utility of GA in the elderly cancer patient population have emerged only recently (5) and should be the focus of future studies.

Although chronological age is a poor descriptor of heterogeneity in the aging process, the clinical scenario analysis revealed that the oncologists in southwestern China were more influenced than the geriatricians by chronological age. A similar result $(3,6-8)$ was found in other countries. Impact factors might be the following: 1) the uncertainty among oncologists of the effects of comorbidity and reduced physiologic reserve on the tolerance to treatment (9), and 2) the differences in professional perceptions of value between the oncologists and geriatricians (10), especially when assessing life expectancy and the overall effectiveness of treatments for cancer patients (8). If the physician believes that an elderly patient will always present with comorbidities, functional disabilities, and poor tolerance to anti-tumor therapy, then this physician will be more likely to prefer a less aggressive treatment plan (7). However, increasing evidence indicates that functional elderly cancer patients can tolerate and benefit from anti-cancer therapies $(11,12)$. If this healthy, aging population is treated similarly to a population with poor tolerance, then the healthy aging patients will not receive the most appropriate care. Again, our results revealed that chronologic age alone is a poor descriptor of heterogeneity in the aging process.

In clinical practice, most of the surveyed oncologists had never cooperated with a geriatrician, which is similar to previously reported findings $(1,13,14)$. However, many studies have found that cooperation among the doctors in these two specialties improves the quality of medical care provided to elderly cancer patients (1517). A majority of the current respondents believed that a «lack of knowledge regarding geriatric diseases» hindered the development of treatment decisions for elderly cancer patients. All of the respondents believed 
that the establishment of a multidisciplinary management team for elderly cancer patients is feasible. Therefore, the establishment of a cooperation model with GA based on a multi-disciplinary team could be necessary and feasible in southwestern China.

The sample size of this study was relatively small, and the respondents were primarily recruited from large public hospitals located in major cities. Moreover, although we defined geriatric concepts such as geriatric syndrome and comorbidity carefully in the survey, the geriatricians and oncologists did have different perceptions of these concepts, which might have produced imprecise results.

Nevertheless, as the first survey conducted among doctors regarding the clinical diagnosis and treatment of elderly cancer patients in China, the study identified some important differences between the two specialties and provided clues for improving health care in the elderly population from the perspective of physicians. In addition, knowledge of geriatrics was disseminated among the oncologists and geriatricians through this survey; this laid a foundation for future cooperation among the physicians in these specialties.

Disclosures and Conflict of Interest Statements: The authors have no conflicts of interest to disclose.

Author Contributions: Concept and design: L.X. Wang, Z.M. Meng, J. Li, S. Wang. Data collection:L.X. Wang, B. Xiang, S.S Nie, Q.Q Sun, H.Qin, Y.L. Gong, C. Yi, C.C. Pan, Y.X. Yang. Quality control of data and algorithms: J. Li, S. Wang. Analysis and interpretation: J. Li, S. Wang. Statistical analysis:L.X. Wang, Z.M. Meng, J. Li, S. Wang. Manuscript writing and approval: L.X. Wang, Z.M. Meng, J. Li, S. Wang. Manuscript editing and review:J. Li, S. Wang.

Acknowledgements: This work was supported by a grant from the Science and Technology of Sichuan Province of China (2010FZ0047).

Ethical Standards: The experiments comply with the current laws of the country

\section{References}

1. Dale W, Mohile SG, Eldadah BA, Trimble EL, Schilsky RL, Cohen HJ, et al. Biological,Clinical, and Psychosocial Correlates at the Interface of Cancer and Aging Research. J Natl Cancer Inst 2012;104:581-589

2. Wildiers H, Heeren P, Puts M, Topinkova E, Janssen-Heijnen ML, Extermann $\mathrm{M}$, et al. International society of geriatric oncology consensus on geriatric assessment in older patient with cancer. Journal of Clinical Oncology, 2015;32(24): 2595-2603

3. Foster JA, Salinas GD, Mansell D, Williamson JC, Casebeer LL. How does older age influence oncologists' cancer management? Oncologist 2010; 15:584-592

4. Bouchardy C, Rapiti E, Blagojevic S, Vlastos AT, Vlastos G. Older female cancer patients: Importance, causes, and consequences of under-treatment. J Clin Oncol 2007; 25:1858-1869

5. Puts MT, Hardt J, Monette J, Girre V, Springall E, Alibhai SM. Use of geriatric assessment for older adults in the oncology setting: A systematic review. J Natl Cancer Inst 2012;104:1133-1163

6. Pang A, Ho S, Lee SC. Cancer physicians' attitude towards treatment of the elderly cancer patient in a developed Asian country. BMC Geriatr 2013; 16 : 13-35.

7. Protière C, Viens $\mathrm{P}$, Rousseau F, Moatti JP. Prescribers' attitudes toward elderly breast cancer patients. Discrimination or empathy? Crit Rev Oncol Hematol 2010; 75:138-150.

8. Blank TO, Bellizzi KM. A gerontologic perspective on cancer and aging. Cancer2008; 112 (Suppl11):2569-2576.

9. Wan-Chow-Wah D, Monette J, Monette M, Sourial N, Retornaz F, Batist G, et al. Difficulties in decision making regarding chemotherapy for older cancer patients: A census of cancer physicians. Crit Rev Oncol Hematol 2011; 78:4558.

10. Sifer-Rivière L, Girre V, Gisselbrecht M, Saint-Jean O. Physicians' perceptions of cancer care for elderly patients: A qualitative sociological study based on a pilot geriatric oncology program. Crit Rev Oncol Hematol 2010; 75:58-69.

11. Langer CJ. Neglected and underrepresented subpopulations: elderly and performance status 2 patients with advanced-stage non-small-cell lung cancer. Clin Lung Cancer, 2006;7( Suppl 4):S126-S137.

12. Muss HB, Berry DA, Cirrincione CT, Theodoulou M, Mauer AM, Kornblith $\mathrm{AB}$, et al. Adjuvant chemotherapy in older women with early-stage breast cancer. N Engl J Med 2009; 360:2055-2065.

13. Puts MT, Girre V, Monette J, Wolfson C, Monette M, Batist G, et al. Clinical experience of cancer specialists and geriatricians involved in cancer care of older patients: A qualitative study. Crit Rev Oncol Hematol 2010; 74:87-96.

14. Gosney M. Contribution of the geriatrician to the management of cancer in older patients. Eur J Cancer2007; 43:2153-2160.

15. Rodin MB, Mohile SG. The geriatrics and oncology dialogues. J Am GeriatrSoc2007; 55:1670-1671.

16. Monfardini S, Aapro M. Cancer treatment in the elderly: the need for a better organization. Ann Oncol2007; 18:1283-1284.

17. Terret C, Zulian GB, Naiem A, Albrand G. Multidisciplinary approach to the geriatric oncology patient. J Clin Oncol 2007; 25:1876-1881.

\section{Appendix}

Questionnaire for doctors treating elderly patients with cancer

1. Gender: $\quad 1 \square$ Male

2. Age: $\quad$ years old

3. Education: $1 \square$ Ph.D. $\quad 2 \square$ Master's $\quad 3 \square$ Bachelor's $4 \square$ College $5 \square$ Vocational school

4. You are working in a:

$1 \square$ Hospital with professional teaching and training

$2 \square$ Public hospital of the first class at grade 3

$3 \square$ Public hospital of the first class at grade 2

$4 \square$ Cancer hospital

$5 \square$ Elderly hospital

$6 \square$ Other

5 . The medical specialty you are currently engaged in:

$1 \square$ General internal medicine

$2 \square$ Geriatrics

$3 \square$ Various internal medicine subspecialties

$4 \square$ Oncology

$5 \square$ Other

6. How many of your patients are elderly people ( $>65$ years old) with cancer?

$$
\begin{aligned}
& 1 \square \text { less than } 10 \% \quad 2 \square 10 \%-30 \% \quad 3 \square 30 \%-50 \% \\
& 4 \square 50 \%-70 \% \quad 5 \square \text { more than } 70 \%
\end{aligned}
$$

7. In your opinion, the overall current treatment situation for elderly patients ( $>65$ years old) with cancer in China is:

$1 \square$ inadequate $2 \square$ appropriate $3 \square$ excessive

4 口 unclear

Please select the top three reasons for your answer above.

* Comorbidity: the simultaneous appearance of two or more psychiatric or physical illnesses. \#Physical function disability, which is composed of more than one dependence of ADL (dressing, eating, ambulating, toileting, hygiene) and IADL (shopping, housework, accounting, food preparation, transportation)

8. Anti-tumor treatment plans for your elderly patients (i.e., $>65$ years old) with cancer are primarily made by:

1 our medical group integrated by both geriatrics and oncologist

\begin{tabular}{|l|l|l|}
\hline Reason & Under-treatment & Over-treatment \\
\hline Physician experience & & \\
\hline Patient personal choice & & \\
\hline Opinion of family members & & \\
\hline Comorbidity* & & \\
\hline physical function disability $\#$ & & \\
\hline $\begin{array}{l}\text { Inadequate reserves of physiological } \\
\text { function }\end{array}$ & & \\
\hline insufficient social support & & \\
\hline Life expectancy & & \\
\hline
\end{tabular}




\section{$2 \square$ oncologists \\ $3 \square$ geriatrics}

9. Have you had ever concerned about physical function disability; comorbidity; mental/cognitive impairment; mood disorder; visual/hearing impairments; nutritional status; caregiver availability; family economy and environment; and patient's willingness for treatment among your elderly patients ( $>65$ years old) with cancer?

$1 \square$ Yes (please write them as following: $2 \square \mathrm{No}$

10. Did you consult a geriatrician/oncologist to co-assess the health status of an elderly patient (i.e., $>65$ years old) with cancer before you created a treatment?

$$
1 \square \text { Yes } 2 \square \text { No }
$$

The frequency with which you cooperate with a geriatrician/ oncologist is:

$$
\begin{array}{ll}
1 \square \text { Never } & 2 \square \text { Occasionally } \\
3 \square \text { In most cases } & 4 \square \text { Always }
\end{array}
$$

11. Which of the following options should be considered carefully when creating a treatment plan for an elderly patient ( $>65$ years old) with cancer?

Please select the five most important factors in your opinion, in descending order.

○ physical condition (dependence of ADL and/or IADL); 0 education level; $\bigcirc$ type of cancer; $\bigcirc$ stage of tumor; $\bigcirc$ current comorbidity; $\bigcirc$ mental/cognitive $\circ$ emotional status; $\bigcirc$ visual/ hearing impairments; $\bigcirc$ nutritional status; $\bigcirc$ income of the patient; $\bigcirc$ caregiver availability; $\bigcirc$ family environment; $\bigcirc$ patient's understanding of the disease; $\bigcirc$ patient's willingness for treatment; $\bigcirc$ opinion of family members

12. Assume that a"healthy" (no comorbidity or comorbidity are not life-threatening-independence of ADL and IADL) old patient with cancer (early stagy) is consultant by you; Please mark the following treatment strategy that you prefer (you may only choose one).

13. Which of the following conditions do you think are the most common factors that affect anti-tumor program development? Please select the five most important factors in your opinion, in descending order.

o chronic heart failure; 0 coronary artery disease; 0 hypertension; $\bigcirc$ type 2 diabetes; $\bigcirc$ chronic obstructive pulmonary disease (COPD) or other chronic respiratory diseases; $\bigcirc$ digestive diseases; $\circ$ liver and kidney dysfunction;

\begin{tabular}{|l|l|l|l|}
\hline Treatment option & $65-74$ & $75-84$ & $\geq 85$ \\
\hline Standard anti-tumor therapy & & & \\
\hline Adjusted/reduced anti-tumor therapy & & & \\
\hline Supportive symptomatic treatment & & & \\
\hline
\end{tabular}

$\bigcirc$ moderate-to-severe osteoporosis; $\bigcirc$ transient ischemic attack (TIA) or cerebral infarction o dementia

14. Do you think that presented incontinence, mobility barriers, and falling can interactive with the toxic side effects of radioand chemotherapy among elderly patients with cancer?

YES NO

15. Do you think that presented cognitive impairments/mood disorders can interactive with the toxic side effects of radio- and chemotherapy among elderly patients? YES NO

16. Do you think that presented malnutrition can interactive with the toxic side effects of radio- and chemotherapy among elderly patients with cancer? YES NO

17. Do you think that applying a multidisciplinary approach (including geriatrics and other medical specialties) to the management of elderly patients ( $>65$ years old) with cancer is feasible? YES NO

If you do not believe this approach is feasible, why? (Circle the 3 most important reasons)

$1 \square$ Lack of awareness

$2 \square$ Not enough geriatric specialists available

$3 \square$ Not enough trained health professionals

$4 \square$ Relatively time-consuming

$5 \square$ Feasibility depends on the institutional system of the hospital

$6 \square$ Might delay patient treatment

18. What do you think is the most significant current barrier regarding the development of a clinical treatment program for elderly patients ( $>65$ years old) with cancer? (You may choose more than one answer)

$1 \square$ Lack of geriatric knowledge

$2 \square$ Elderly patients trust the oncologist more than other doctors $3 \square$ Greater vulnerability among elderly patients

$4 \square$ Other 